\title{
Slow and Steady Keeps Them in the Race: Metronomic Therapy in Children With Cancer
}

\author{
SidHaRTH TotadRI \\ Pediatric Hematology-Oncology Unit, Department of Pediatrics, Christian Medical College and Hospital, Vellore, India. \\ totadri86@yahoo.com
}

$\mathrm{S}$ urvival in childhood cancer has improved to approximately $80 \%$ in high-income countries (HIC) [1]. This success story is attributable to advances in diagnosis and risk stratification, and the protocolized administration of cytotoxic therapy $[1,2]$. However, the survival lags by $50 \%$ in low-income and low-and middle-income countries (LMIC) [1]. The road blocks faced by children with cancer in LMIC include resource constraints, a delayed presentation with advanced disease, treatment abandonment, malnutrition, and increased treatment-related toxicity. Treatment protocols that have been established and validated in HIC are not designed to address the challenges prevalent in LMICs [2]. Malignancy accounts for approximately $1 \%$ of deaths in children aged 5-14 years in India [3]. Novel Cost-effective strategies to treat children with cancer are of immense importance in LMICs such as India.

Conventionally, oncological trials have focused on the eradication of the malignancy and the reduction of relapse. With the best of infrastructure, a realistic possibility of a cure in every child with cancer is not conceivable. Five-year overall survival reported in highrisk malignancies such as acute myeloid leukamia, soft tissue/bone sarcomas and neuroblastoma; is markedly lesser when compared to malignancies such as Hodgkin lymphoma, Wilms tumor and germ cell tumors [4]. Survival is notably inferior in metastatic cancers and cancers that are refractory to therapy, or relapse following completion of therapy. Salvage treatment for such cancers is resource-intensive and toxic, and therefore often impractical in LMICs [5].

Metronomic therapy is an alternative paradigm in the management of children with cancer [5]. The approach involves the prolonged administration of chemotherapeutic agents in low, minimally toxic doses with no prolonged drug-free breaks [6]. Further, repurposed noncytotoxic drugs, e.g. celecoxib, thalidomide and valproate, are incorporated into metronomic protocols [7]. In comparison, standard chemotherapy regimens utilize maximally tolerated doses (MTD) of cytotoxic drugs administered over a definite time [6]. Chemotherapy at MTD directly targets the cancer cells, which have an inherent tendency to develop mechanisms of resistance (akin to bacteria treated with antibiotics) [8]. Therapy at MTD necessitates breaks to allow recovery from toxicity, which further facilitates tumor cell proliferation [6]. Alternatively, metronomic therapy attempts to collapse the house by breaking the scaffold. That is, it targets the endothelial cells in the tumor microenvironment by anti-angiogenic mechanisms $[6,8]$. Additionally, metronomic therapy attempts to switch on the natural immune surveillance mechanisms against the malignant clone and induce tumor cell dormancy $[6,8]$. The prolonged oral maintenance therapy in acute lymphoblastic leukemia and the recent evidence favoring maintenance therapy in high-risk rhabdomyo-sarcoma stand testimony to the impact of a metronomic approach on outcome in pediatric malignancies $[9,10]$. Web Table I lists recent Indian studies which have demonstrated the feasibility and utility of metronomic therapy in children with high-risk cancers. Low cost, minimal toxicity, homebased intake of oral drugs, and a reduction in the need to travel to the hospital and admission comprise the selfevident benefits of metronomic therapy in LMICs $[5,6]$.

Although metronomic therapy appears simple and attractive, there are caveats which need to be addressed. Standard chemotherapy regimens are ratified by randomized trials. Phase III trials, response criteria such as radiological remission in solid malignancies and lymphomas, minimal residual disease assessment in leukemia and outcomes such as disease-free survival may not be relevant in patients on a metronomic regimen $[5,11]$. Pharmacokinetic studies to optimize drug doses, identification of beneficiary disease subgroups and biological markers for response assessment constitute areas that merit research in the metronomic field [11]. This issue of Indian Pediatrics carries an important study in this regard. Pramanik, et al. [12] performed a placebocontrolled randomized trial of metronomic therapy in children with progressive extracranial solid malignancies. 
The clinical aspects of the study were published previously and are briefly described in Web Table I [12]. The current study evaluated specific biomarkers in the same patient cohort. The authors examined the baseline and subsequent levels of vascular endothelial growth factor (VEGF) a proangiogenic cytokine, and thrombospondin-1 (TSP-1), an anti-angiogenic cytokine [13]. Although the study concluded that these were not reliable biomarkers for assessment of response to metronomic chemotherapy, some interesting trends were illustrated. The baseline VEGF levels were lower in responders. TSP-1 decreased in responders and increased in non-responders in the metronomic arm. A similar finding was not observed in the placebo arm. The results reaffirm the influence of metronomic treatment on angiogenesis. The study opens new vistas for research in metronomics. For instance, biomarkers such as VEGF levels may show promise as surrogates for identifying patients who would benefit with a metronomic approach. Since the effects of metronomic therapy encompass multiple pathways, the authors rightly state in their discussion that a broader spectrum of circulating biomarkers needs to be studied to yield clinically relevant indicators [13].

A survey of pediatric oncology physicians working in LMIC revealed a strong belief that the use of metronomic therapy is likely to increase with time [14]. An overwhelming majority expressed interest to participate in international studies and registries [14]. Research in metronomic therapy can fill many lacunae, if not all, in the treatment armamentarium for children with cancer.

Competing interests: None stated; Funding: None.

\section{REFERENCES}

1. Lam CG, Howard SC, Bouffet E, Pritchard-Jones K. Science and health for all children with cancer. Science. 2019;363:1182-6.

2. Howard SC, Davidson A, Luna-Fineman S, Israels T, Chantada G, Lam CG, et al. A framework to develop adapted treatment regimens to manage pediatric cancer in low-and middle-income countries: The Pediatric Oncology in Developing Countries (PODC) Committee of the International Pediatric Oncology Society (SIOP). Pediatr Blood Cancer. 2017;64.

3. Causes of Death Statistics. Census of India Website. Available from: http://censusindia.gov.in/vital_statistics/ causesofdeath.html. Accessed March 3, 2020.

4. Arora RS, Eden TOB, Kapoor G. Epidemiology of childhood cancer in India. Indian J Cancer. 2009;46:264-73.

5. André N, Banavali S, Snihur Y, Pasquier E. Has the time come for metronomics in low-income and middle-income countries? Lancet Oncol. 2013;14:e239-48.

6. André N, Banavali S, Fousseyni T, Pasquier E.
Metronomic chemotherapy in pediatric oncology: A way forward for low-income countries? In: Oncopedia. St. Jude Cure4Kids: An International Medical Education Website and Online Collaboration Center. Available from: https://www.cure4kids.org/.Accessed February 23, 2020.

7. Rumore MM. Medication repurposing in pediatric patients: Teaching old drugs new tricks. J Pediatr Pharmacol Ther. 2016;21:36-53.

8. Kareva I, Waxman DJ, Lakka Klement G. Metronomic chemotherapy: An attractive alternative to maximum tolerated dose therapy that can activate anti-tumor immunity and minimize therapeutic resistance. Cancer Lett. 2015;358:100-6.

9. Andre N, Cointe S, Barlogis V, Arnaud L, Lacroix R, Pasquier E, et al. Maintenance chemotherapy in children with ALL exerts metronomic-like thrombospondin-1 associated anti-endothelial effect. Oncotarget. 2015;6: 23008-14.

10. Bisogno G, De Salvo GL, Bergeron C,GallegoMelcón S, Merks JH, Kelsey A, et al.Vinorelbine and continuous lowdose cyclophosphamide as maintenance chemotherapy in patients with high-risk rhabdomyosarcoma (RMS 2005): A multicentre, open-label, randomised, phase 3 trial. Lancet Oncol. 2019;20:1566-75.

11. Pantziarka P, Hutchinson L, André N, Benzekry S, Bertolini F, Bhattacharjee A, et al. Next generation metronomic chemotherapy-report from the Fifth Biennial International Metronomic and Anti-angiogenic Therapy Meeting, 6-8 May 2016, Mumbai. Ecancermedicalscience. 2016;10:689.

12. Pramanik R, Agarwala S, Gupta YK, Thulkar S, Vishnubhatla S, Batra A, et al. Metronomic chemotherapy $v s$ best supportive care in progressive pediatric solid malignant tumors: A randomized clinical trial. JAMA Oncol. 2017;3:1222-7.

13. Pramanik R, Tyagi A, Agarwala S, Vishnubhatla S, Dhawan D, Bakhshi S. Evaluation of vascular endothelial growth factor (VEGF) and thrombospondin-1 as biomarkers of metronomic chemotherapy in progressive pediatric solid malignancies: A randomized study. Indian Pediatr. 2020;57:508-11.

14. Revon-Rivière G, Banavali $\mathrm{S}$, HeississenL, Gomez Garcia $\mathrm{W}$, Abdolkarimi B, Vaithilingum $\mathrm{M}$, et al. Metronomic chemotherapy for children in low-and middle-income countries: survey of current practices and opinions of pediatric oncologists. J Glob Oncol. 2019;5:1-8.

15. Banavali S, Singh R, Prasad M, Arora B, Nahar A, Vora T, et al. Is there a role for a novel maintenance therapy in pediatric patients with AML? In: 43rd Congress of the International Society of Paediatric Oncology (SIOP) 2011 Auckland, New Zealand, 28th-30th october, 2011. Abstract PB015. Pediatr Blood Cancer. 2011;57:770.

16. Devadas SK, Banavali S. Retrospective analysis of outcomes of patients with relapsed, refractory and metastatic sarcomas who have received metronomic chemotherapy. Gulf J Oncol. 2019;1:22-8. 